Penser et pratiquer la formation aujourd'hui : Mise en perspective des approches psychosociales et ergonomiques de la formation

Daniel Faulx

Université de Liège

Faculté de Psychologie et des Sciences de l'Education

Unité d'Apprentissage et de Formation continue des Adultes (UAFA)

Boulevard du Rectorat, 5/B32

4000 LIEGE

Belgique

Tél. : +3243664675

Fax : + 3243662944

Courriel : Daniel.Faulx@ulg.ac.be

Lucie Petit

Université des Sciences et Technologies de Lille 1

Faculté des Sciences de l'éducation

Bureau 203, 2ième étage, bâtiment B6, Cité Scientifique,

59000 Villeneuve d'ascq

France

Courriel : Lucie.petit@univ-lille1.fr 


\title{
Penser et pratiquer la formation aujourd'hui : Mise en perspective des approches psychosociales et ergonomiques de la formation
}

\section{RESUME}

Le domaine de la formation est actuellement dominé, tant du point de vue scientifique que de celui des pratiques, par deux grands courants: le courant psychosociologique et le courant ergonomique. Si l'apport de l'un comme de l'autre est incontestable, on constate une grande méconnaissance entre ces deux courants, ainsi que l'absence d'un dialogue et d'une mise en perspective scientifique. Cette contribution propose d'initier cette démarche, à la fois dans l'optique de développer les pratiques des formateurs - qu'il s'agisse de professionels de la formation ou de personnes appelées à jouer ce rôle ponctuellement dans leur carrière -, mais aussi d'aider les commanditaires de formation à mieux orienter leurs choix de formation grâce à une meilleure compréhension des deux approches.

De cette mise en perspective réalisée à partir tant des travaux historiques que des recherches les plus récentes, il ressort d'abord plusieurs points communs. (1) Une volonté de développer de nouveaux modes d'apprentissage, nourris par des recherches scientifiques, et qui rompent avec les approches scolaires. (2) Un lien fort établi entre théorie et action, avec des pratiques qui articulent la pratique et la connaissance, l'intervention sur l'organisation et l'apprentissage individuel. (3) Une vision de l'apprentissage comme résultant de l'action combinée avec la réflexion sur l'action.

En contraste avec ces fondements communs, plusieurs points de divergences se prêtant à de fructueux échanges, sont identifiés. (1) Un ancrage prioritaire sur le groupe pour les approches psychosociologiques, sur le travail pour les approches ergonomiques. (2) Une centration sur les aspects plutôt fonctionnels et spécifiques du travail pour l'approche ergonomique, plutôt sur les aspects relationnels et transversaux pour l'approche psychosociologique. (3) Quatre points sur lesquels des échanges de techniques seraient profitables. (4) Une controverse sur la question du lien - nécessaire ou non - entre travail et formation. En prenant acte de la complémentarité des deux approches (à la fois compatibles et différentes), il apparaît utile d'entreprendre un dialogue tant dans une optique de lisibilité des pratiques de formation en organisation que d'efficacité. Un tel constat invite donc les formateurs à intensifier leurs échanges, et les commanditaires à mobiliser les apports conjoints des deux approches afin de développer au mieux les ressources humaines de leurs organisations.

\section{Mots-clés : analyse de l'activité, animatique des groupes, formation, ergonomie, psychosociologie}

\begin{abstract}
Faced with new challenges from globalisation, technological changes and demographic change, organisational training take on today a crucial importance in the development of human resources in organisations. This area is at present dominated from both a scientific and practical viewpoint by two major currents: a psychosociological current and an ergonomic current. The contributions of each in organisational human resources development are
\end{abstract}


unquestionable, but we have observed significant levels of misunderstanding between the two, as well as an absence of dialogue and scientific objectivity. The present contribution proposes to initiate this dialogue, with an eye toward developing training practices, and aiding those who seek to train personnel in orienting their choices of training, thanks to a better understanding of both main approaches.

From this perspective, based as much on historical research as on more recent work, several points in common are apparent. (1) There is a desire to develop new learning strategies which draws substance from scientific research, and which constitutes a departure from school-based approaches. (2) There is a strong link between theory and action, together with practices that combine practice and knowledge. (3) A vision of learning as the result of action combined with reflection on action. (1) A concentration on groups from psychosociological approaches, as opposed to a concentration on work from ergonomic perspectives. (2) A concentration on functional and specific aspects of work in the ergonomic approach, and emphasis on relational and transversal aspects in the psychosociological approach. (3) Four points concerning which technical exchanges would be profitable. (4) A major controversy on the question of whether the link between work and training is necessary or not. By becoming aware of the complementarity of the two approaches (compatible and different), we become convinced that it is essential to begin a dialogue in order to make organisational training practices more understandable and more effective.

Key words : animatics, ergonomics, psychosociology, training 


\section{Les deux mondes de la formation : apports, différences et complémentarité.}

\section{Mise en perspective des approches psychosociales et ergonomiques de la formation}

Cet article est le fruit d'une collaboration entre deux universités (Liège et Lille 1), deux revues (RI-IR et les CIPS) et deux auteurs, porteurs chacun, à l'origine, d'une vision différente de la formation.

Rarement, ces visions ont l'occasion de dialoguer tant les lieux de publication, de commnication et de formation épousent ces deux approches. C'est pourquoi nous proposons de mettre à disposition le fruit de cette réflexion comparative. Enthousiaste à cette idée, les CIPS nous ont invités à reprendre le propos d'un article publié dans la revue canadienne Relations Industrielles (Faulx \& Petit, 2010), dont l'objet est la comparaison et la mise en persepctive des approches psychosociales et ergonomiques de la formation. Cette publication commune prolonge ainsi cette intention de partage de réflexions autour de deux conceptions très différentes de la formation que l'on rencontre actuellement.

\section{Introduction : quels enjeux autour de la formation aujourd'hui ?}

La formation des adultes se trouve aujourd'hui au coeur d'enjeux économiques et sociaux considérables (Merle, 2006 ; Voisin, 2004). Il apparaît donc essentiel que formateurs, usagers et commanditaires de la formation identifient de quelle manière et avec quels cadres méthodologiques et conceptuels elle entend remplir le rôle dont elle est investie aujourd'hui, et plus spéciquement qu'ils soient en mesure de reconnaître, distinguer et mettre en perspective les deux courants principaux qui structurent ce domaine de recherche et d'action : les approches psychosociologiques (Maisonneuve, 2005) et les approches ergonomiques (Falzon \& Teiger, 2004).

\section{Cadrage général : deux courants}

Historiquement, l'émergence de ces deux courants - que nous appelerons approches psychosociales de la formation (APF) et approches ergonomiques de la formation (AEF) date d'une soixantaine d'années. En effet, bien que la pratique de la formation en tant qu'activité humaine constitue une entreprise très ancienne, sa structuration en champ de recherche, d'intervention et d'action s'est constituée essentiellement au lendemain de la seconde guerre mondiale (Mucchielli, 2006) - exception faite de quelques travaux précurseurs comme ceux de Lindeman (1926) - , période au cours de laquelle sont apparues les premières tentatives d'intégrer concepts, notions et résultats de recherche dans le domaine (Knowles, 1990).

D’une part, dans la lignée des travaux de Lewin (Faucheux, 1959 ; Lewin, 1945 ; Lewin \& Faucheux, 1967 : Michelot, 2003), la psychosociologie, depuis la période 1940-1950 et de manière accrue depuis les années 60 (Tilman \& Grootaers, 2006), a fourni un ensemble de cadres de références, de pratiques, d'éléments idéologiques et de positionnements épistémologiques qui marquent encore aujourd'hui fortement les pratiques quotidiennes de l'ensemble des formateurs d'adultes (Jobert, 2003), et ont largement pénétré le milieu 
organisationnel au cours des quarante dernières années (Goguelin, Cavozzi, Dubost, Enriquez, 1971; Maisonneuve, 2005). D'autre part, l'ergonomie, dans la suite des travaux d'Ombredanne et Faverge (1955), est devenue une référence majeure pour la recherche en formation depuis les années 1980 (Clot, Daniellou, Jobert, Mayen, Olry \& Schwartz, 2005), et occupe une place incontournable dans les pratiques formatives en organisation aujourd'hui (Parage, 2008), dotant également les formateurs de cadres méthodologiques, épistémologiques et théoriques qui leur ont permis de rapprocher la formation du monde du travail et des organisations (Olry \& Parage, 2008).

Or, ces deux cadres de pensée et d'action se parlent peu, pour ne pas dire pas du tout. Plusieurs symptômes attestent de leur ignorance réciproque. Tout d'abord, les lieux de publications sont cloisonnés: les travaux théoriques et empiriques sur la formation des adultes d'inspiration psychosociologique sont publiés dans des revues comme les Cahiers Internationaux de Psychologie Sociale (Delhez, 1999 ; Faulx \& Delvaux, 2007 ; Patin, 2005) alors que les travaux situés dans le courant ergonomique sont publiés, par exemple, dans Relations Industrielles (Bellemare, Montreuil, Marier, Prévost \& Allard, 2001 ; Fournier, 2005 ; Lamonde \& Montreuil, 1995, Montreuil \& Bellemare, 2001, Saint-Vincent, Lortie \& Chicoine, 2001). Les études qui s'appuient sur les deux courants sont rares - exception notable, l'article de Clot (2006) qui aborde les liens entre clinique du travail et psychosociologie clinique -, et les ouvrages de référence eux-mêmes n'échappent pas à ce clivage. A titre d'exemple, on notera l'absence de concepts-clés issus de «l'autre approche » dans deux ouvrages majeurs de synthèse : dans l'index du Vocabulaire de Psychosociologie (Barus-Michel, Enriquez \& Lévy, 2003), aucune entrée à activité, à analyse de l'activité, à ergonomie, alors que plusieurs chapitres sont consacrés à la formation; dans le Traité des Sciences et Technique de la Formation (Carré \& Caspar, 2004), aucune entrée à dynamique des groupes, à animation de groupes, à psychosociologie. Enfin, les centres de formation de formateurs et les pratiques des formateurs eux-mêmes semblent épouser cette dualité.

Pour entreprendre cette mise en perspective entre approche ergonomique et approche psychosociologique de la formation, nous identifierons d'abord les points de convergences, qui attestent de préoccupations communes, puis les objets d'échange (de rapprochement, de confrontation, de conflit, de débat), en nous basant à la fois sur les travaux historiques qui fondent l'identité des courants et sur les recherches les plus récentes qui en portent les développements actuels.

Avant de réaliser cette approche comparative, il est utile de préciser que, si cette distinction revêt à nos yeux une valeur heuristique importante, elle présente comme toute forme de classification une vision toujours plus tranchée que la finesse des constructions et des intégrations personnelles de chaque formateur ou chercheur.

C'est pourquoi nous parlons au pluriel des approches psychosociales et des approches ergonomiques de la formation. Nous aurons l'occasion au cours du développement de cette mise en perspective de mentionner et commenter plusieurs courants au sein de chaque approche. D'autre part, si ces deux courants constituent une base conceptuelle et méthodologique déterminante, il faut également avoir à l'esprit que les pratiques et épistémologies de formation s'articulent avec d'autres domaines. Comme le rappelaient récemment Astier et Baroth (2010), «psychosociologie et ergonomie n'ont été dominantes dans le champ de la formation que combinées à d'autres approches, que l'on peut peut-être évaluer comme de moindre importance, mais qui ont assuré une part de leur succès opérationnel» (p. 3). Ces auteurs citent notamment les apports des sciences sociales en 
général aux pratiques de formation, et en particulier la sociologie des organisations, le domaine de l'ingénierie sociale. Les auteurs rappellent aussi que des notions transversales peuvent connaître un certain succès pour les deux approches comme celles de compétences et de professionnalisation ou encore celle d'analyse des besoins.

\section{Points de convergences}

\subsection{Une volonté commune de développer de nouveaux modes d'apprentissage}

Le premier point commun qui relie les approches psychosociologiques et les approches ergonomiques de la formation est que l'une et l'autre ont développé un discours, des théories, des recherches et des outils orientés vers la formation des adultes à partir de disciplines dont ce n'était pas nécessairement le projet princeps. Ainsi, du côté de la psychosociologie, il existe un grand nombre de disciplines (psychologie sociale, dynamique des groupes, animatique des groupes, psychologie des interactions sociales et socio-cognitives, psychosociologie clinique, ...) dont les développements ont donné lieu à des applications dans le domaine de la formation. Du côté de l'ergonomie, on compte aussi une variété d'approches (analyse de l'activité, clinique de l'activité, analyse du travail, psychologie ergonomique, ergonomie cognitive, ...) qui ont donné lieu à des indications sur la manière de créer des ingénieries de formation efficaces.

Il s'agit donc de courants qui, au départ, ne se constituent pas comme des approches pédagogiques au sens strict, mais au sein desquels les préoccupations relatives à l'apprentissage des adultes sont présentes depuis assez longtemps. Ainsi, dès les années 40, les travaux de Lewin se sont penchés sur les mécanismes de l'apprentissage humain, sous l'appellation de "rééducation" (Lewin, 1945) et, du côté de l'ergonomie, Ombredanne et Faverge (1954) se sont intéressés de près, dans leur ouvrage sur l'analyse du travail, aux questions de formation. Très vite, à partir de l'un et l'autre courant, se sont développées des pratiques et théories pédagogiques visant les adultes en milieu organisationnel, dont les plus emblématiques sont, pour les psychosociologues, les pédagogies de groupe (Meirieu, 1987) et pour les ergonomes, la didactique professionnelle (Pastré, Mayen \& Vergnaud, 2006).

Cette volonté d'appliquer des approches scientifiques aux questions de formation ne constitue pas le seul aspect de ce socle commun. Les démarches pédagogiques et formatives issues des deux approches se sont bâties sur un principe d'opposition (Tourraine, 1973) similaire : la remise en question du modèle scolaire, avec une démarcation de l'enseignement traditionnel et de son mode de transmission des connaissances. Ainsi, du côté de la psychosociologie, la figure de l'animateur se constitue en rupture avec les pédagogies traditionnelles (Jobert, 2003), débouchant sur plusieurs courants pédagogiques dont le point commun est la perspective d'une action libératrice par des pédagogies de l'expérimentation ou de l'expérienciation (Tilman \& Grootaers, 2006). Du côté de l'ergonomie, la conception éthique et épistémologique se différencie également du modèle scolaire traditionnel en proposant la figure de l'intervenant ou de l'intervenant-formateur, qui se conçoit comme un expert parmi d'autres, coopérant avec les apprenants en tant que co-producteur de connaissances, dans une démarche de co-construction sociale (Teiger \& Lacomblez, 2005). Le courant, et l'expression même, d' "ergonomie participative », qui mêle recherche-action et formation (St-Vincent, Lortie \& Chicoine, 2001) en est un exemple particulièrement parlant.

De cette prise de distance avec le système scolaire, il résulte dans les deux cas des postulats éthiques, méthodologiques et épistémologiques qui conduisent à un nouveau rapport au 
savoir et au pouvoir. Quand des psychosociologues concluent à l' «incomplétude des savoirs théoriques » et à la nécessité de construire des savoirs par la pratique (De Visscher, 2001) et que des ergonomes distinguent connaissances théoriques et connaissances pour l'action (Vidal-Gomel \& Rogalski, 2007) et affirment la place importante des savoirs construits dans l'action (Pastré, 2007), ils se rejoignent sur un point : la théorie enseignée du haut vers le bas du magister omniscient à l'élève naïf ne convient pas aux adultes, spécifiquement dans le champ de l'organisation. En reconnaissant la place de la coconstruction dans l'apprentissage, l'importance des représentations des apprenants, la nécessité de l'implication de l'adulte dans son apprentissage, en valorisant l'autonomie de l'apprenant, ou encore en militant pour la reconnaissance de l'expertise ouvrière, formateurs psychosociologues et formateurs ergonomes ont les uns et les autres contribué à donner une place importante aux formés dans le processus formatif. Ils sont en cela les héritiers communs de Lindeman qui, dès 1926, prônait que l'éducation des adultes place au centre de ses préoccupations les besoins, les centres d'intérêts et l'expérience des apprenants, à l'inverse de l'école qui, disait-il, considère les élèves comme des éléments secondaires par rapport aux programmes et aux enseignants.

\subsection{Le lien entre théorie et action}

Un second élément qui rapproche la vision psychosociologique et la vision ergonomique est la liaison entre la connaissance, d'une part, et le changement de l'organisation et du travail d'autre part. Les deux courants se revendiquent d'une épistémologie à la fois compréhensive et transformationnelle. Ainsi, pour Clot, toute analyse de l'activité est une transformation de cette activité qui en fait reculer l'horizon (Clot et al. , 2005 : 154). De manière plus générale, l'enjeu de l'approche ergonomique est de comprendre le travail pour le transformer (Guérin, Laville \& Daniellou, 1997). Les approches ergonomiques de la formation lient ainsi explicitement les aspects d'analyse, d'intervention et de formation (Teiger \& Lacomblez, 2005 ; Bellemare, Montreuil, Marier, Prévost, \& Allard, 2001, 2004).

Les psychosociologues tissent également un lien fort entre compréhension et action, même si l'objet de la transformation porte généralement davantage sur les personnes et sur les relations qui se tissent dans l'organisation que sur le travail lui-même. Il faut rappeler à ce sujet que Lewin n'est pas seulement l'inventeur de la dynamique des groupes moderne, il est aussi un des créateurs de la recherche-action, et défendait donc la double liaison entre la connaissance et l'action (Marrow, 1972; Michelot, 2003; Hess, 1981). Dans sa définition de la psychosociologie clinique, Enriquez (1993) rappelle que, dans cette discipline, il ne peut y avoir de connaissance d'un objet sans intervention sur cet objet. Si l'on admet que la gestion et l'animation des groupes est un des éléments fondamentaux de l'approche psychosociologique en formation (Goguelin, 1987; Maisonneuve, 2005), on rappellera également que depuis ses débuts, l'expression «dynamique des groupes » recouvre un double sens (Faulx, 2008): d'une part, elle désigne l'ensemble des recherches portant sur les phénomènes groupaux (Lapassade, 1971), et, d'autre part, elle rend compte de pratiques et

instruments de formation développés au sein du groupe « restreint » (De Visscher, 2004). La connaissance et l'action, mais aussi l'apprentissage individuel et la transformation de l'organisation sont donc, dans ces deux courants, indisociables.

\subsection{Une liaison entre action, réflexion sur l'action et apprentissage}

Un troisième trait commun tient dans la manière dont ces deux approches opèrent un lien entre l'apprentissage et l'action (ou l'activité). Chacune le dit à sa manière : pour Karolewicz (1998), l'homme est un être empirique: il apprend en faisant. Pour Fournier (2004), l'apprentissage est indissociable de l'activité et l'activité indissociable de l'apprentissage. 
Plus spécifiquement, on peut tracer plusieurs convergences. Tout d'abord, les deux approches reconnaissent une place centrale à l'expérience. C'est pourquoi elles ont l'une comme l'autre développé des méthodes de formation qui, si elles empruntent des voies méthodologiques différentes, prennent acte de l'expérience professionnelle des participants, l'utilisent, la sollicitent et l'intègrent dans les dispositifs. Du côté de la psychosociologie, des pratiques comme les groupes de paroles (Quaderi, 2004), les communautés de pratiques (Wenger, 1998), l'analyse clinique des conduites professionnelles en groupe (Vidal, 2006), les expériences structurées en groupe (Delhez, 1999), les trames expérienciées (De Visscher, 2004) mobilisent l'expérience des participants en situation réelle ou simulée pour générer de l'apprentissage. Du côté ergonomique, l'analyse des pratiques professionnelles (Doyle, 1990 ; Blanchard- Laville \& Fablet, 2000), la didactique professionnelle (Pastré, 2008), l'analyse réflexive collective de l'activité (Gonzalez, 2005) ou encore les nombreuses applications de l'analyse de l'activité à l'ingénierie de formation (Veyrac \& Bouillier, 2011) mobilisent elles aussi l'expérience des apprenants pour construire et mettre en œuvre les dispositifs de formation.

Ensuite, il existe une conviction commune selon laquelle l'apprentissage découle de la mise en lien entre l'action et la réflexion sur l'action. Sur ce point, les deux courants partagent une filiation commune avec les travaux de Dewey (Bourgeois, 2007), qui est l'un des premiers à démontrer que l'expérience joue un rôle important dans la construction des savoirs (Liétard, 2002), mais aussi avec ceux d'Argyris et Schön (Argyris \& Schon, 1974, Argyris, 1995) qui sont à la fois repris en didactique professionnelle (Buysse, 2011) et en psychosociologie de l'apprentissage (De Visscher, 2001). Les deux approches considèrent ainsi qu'apprendre passe non seulement par l'activité (ou l'action), mais également par la réflexion sur cette action (ou activité). Selon les termes de Samurçay et Rabardel (2004), l'activité humaine est à la fois productive, en ce sens qu'elle provoque des transformations sur le monde matériel, social et symbolique, mais également constructive, en ce sens qu'elle provoque des transformations sur les personnes, c'est-à-dire finalement des apprentissages. Cette idée, cruciale dans le courant ergonomique, rencontre les théories de l'apprentissage expérientiel, un concept introduit par Rogers (1969) et qui marque fortement les pratiques psychosociologiques qui en sont largement héritières (Jobert, 2003). Méthodologiquement, cela se transcrit dans des pratiques différentes, mais finalement comparables sur un plan épistémologique. Les psychosociologues insistent sur l'importance de la phase de débriefing (Baiwir \& Delhez, 1979 ; Chamberland \& Provost, 1996 ; Delhez, 1999 ; Piskurich, Beckshi \& Hall, 2000) ou d'élucidation collective (De Visscher, 2004 ; Faulx \& Delvaux, 2007), au cours de laquelle le formateur aide le groupe à faire émerger les éléments essentiels d'apprentissage, et applicables en contexte organisationnels, qui peuvent être retirés de l'expérience. Du côté des approches ergonomiques, Pastré (2005a) insiste sur l'importance de l'analyse réflexive après l'action. Pour lui, on apprend au moins autant en revenant de manière rétrospective sur son action qu'en en observant les effets, d'où l'importance cruciale des activités de débriefing et d'autoconfrontation.

Les deux approches partagent également la volonté de préserver des traces de l'activité des apprenants. Selon Pastré (2005a), pour procéder à cette analyse réflexive rétrospective, il faut disposer de traces autres que des souvenirs. Les psychosociologues et dynamiciens de groupe le rejoignent lorsqu'ils utilisent des grilles d'observation collectées par des participants ou le formateur à des fins d'apprentissage (voir par exemple Landry, 2008).

Enfin, pour conclure ce point sur la liaison entre expérience, action, apprentissage et réflexion sur l'action, on ajoutera que les deux approches sont convaincues que l'activité constructive 
d'apprentissage consécutive à l'action nécessite de faire intervenir un processus social. Cet échange en groupe étant au cœur de la méthodologie des trames expérienciées en groupe (De Visscher, 2004), on ne sera pas surpris de la trouver du côté des approches psychosociologiques. Mais elle est également présente chez les ergonomes de la formation. Pour Pastré (2005a) il est pratiquement impossible de procéder seul à l'analyse de son activité. L'autre joue donc un rôle de médiateur fondamental dans le processus d'apprentissage, y compris sur soi, et le processus social entre les partenaires - qu'il s'agisse du duo formateur/tuteur - apprenant (Kunégel, 2005 ; Zeitler \& Berguerioux, 2007) ou de deux personnes engagées dans un processus de formation, comme par exemple un futur formateur et un futur professionnel (Beckers, 2011) - va constituer un élément déterminant de celui-ci.

\section{Les apports possibles : objets de divergences et d'échanges}

Après avoir identifié un point majeur d'apport de chaque approche, nous nous penchons sur trois questions qui se prêtent à des échanges de point de vue, de techniques ou de concepts.

\subsection{Deux ancrages}

\section{a. L'ancrage groupal : l'apport de la psychosociologie}

Du côté des apports des psychosociologues, incontestablement, la compréhension du groupe sa dynamique, sa gestion et sa contribution à l'apprentissage, son importance dans le travail -, constitue un point d'apport essentiel à la formation en organisation (Abric, Doise, De Visscher, Maisonneuve \& Pain, 1999). Pour les psychosociologues de la formation, le groupe n'est pas simplement un contexte dans lequel le formateur travaille, c'est un outil d'apprentissage. L'énergie du groupe doit être captée pour devenir motrice de la formation (Mucchielli, 2006). Dans la méthode des expériences structurées, c'est par le travail réflexif du groupe sur son activité (groupale et individuelle) que les participants vont être en mesure de faire des apprentissages (Delhez, 1999). Le groupe est vu par les psychosociologues non comme une contingence ou un contexte d'apprentissage mais comme moyen indispensable de cet apprentissage, et parfois, dans le cadre de la dynamique des groupes, comme l'objet même de l'apprentissage. Le travail en équipe, la gestion des groupes, le leadership constituent ainsi des objets privilégiés. Il existe en la matière une quantité énorme de situations d'apprentissages en groupe dans les huit volumes de Pfeiffer et Jones (1983), ainsi que dans chacun des numéros des Cahiers Internationaux de Psychologie Sociale des trente dernières années.

De manière plus large, les psychosociologues ont montré la nécessité, pour que l'apprentissage ait lieu, d'une dynamique groupale favorable à celui-ci. S'il s'agit plutôt une intuition lors des premières applications, ce postulat se verra confirmé par les travaux sur le conflit socio-cognitif (Perret-Clermont, 1996). Les tenants de ce courant considèrent que, le groupe étant le médium de l'apprentissage, le formateur (ou le gestionnaire d'une équipe de travail qui souhaite développer les compétences de ses membres) doit être capable de décoder sa dynamique à la fois pour proposer des situations d'apprentissage adéquate et pour en tirer profit, en se basant notamment sur les stades d'évolution ou de maturité du groupe (SaintArnaud, 1978 ; Tuckman, 1965) et l'identification des processus d'affection, de travail et de pouvoir (Landry, 2008). Il pourra ainsi également sélectionner adéquatement les situations miniatures qui permettent l'apprentissage. On entre ici dans le domaine de l'animatique des groupes, ou science-action de l'animation (Faulx, 2008), un domaine porteur de riches informations sur la manière d'animer un groupe (que ce soit dans la conception initiale supports pédagogiques, expériences structurées - ou dans la conception en cours d'usage - 
attitudes à adopter, manières de réguler les échanges, ... -) (Delhez, 1999, De Visscher, 2001, Faulx, 2007).

Enfin, la pratique de l'élucidation collective ou du débriefing (De Visscher, 2004), cœur de l'activité d'animation des groupes (Delhez, 1999), constitue une spécificité des approches groupales qui mérite toute l'attention: elle permet à l'animateur d'organiser une réflexion métacognitive en groupe (Bourgeois \& Nizet, 1997), aidant les participants à dégager des apprentissages utilisables dans leur vie professionnelle et personnelle (Baiwir \& Delhez, 1979 ; Chamberland \& Provost, 1996). Elle suppose la mise en place d'attitudes spécifiques d'animation qui feront en sorte que le groupe et ses membres pourra être efficacement accompagné dans sa démarche d'apprentissage (Delhez, 1999 ; De Visscher, 2001, 2006). Le groupe constituant l'unité de base en formation (Jobert, 2003), ces techniques et disciplines constituent des apports considérables pour l'ensemble des formateurs et gestionnaires d'équipes.

\section{b. L'ancrage dans le travail : l'apport de l'ergonomie}

Du côté des approches ergonomiques, un apport fondamental réside certainement dans les manières d'approcher, d'observer, de comprendre et d'utiliser le travail en lien avec l'ingénierie de formation. On peut le résumer à partir du postulat «simple et fort » de la didactique professionnelle : la qualité du dispositif de formation sera fonction de la qualité de l'analyse du travail (Pastré, 2005a). L'apport n'est pas seulement méthodologique, avec la diversité des techniques d'analyse du travail qui ont été mises au point par les ergonomes (voir ci-après), mais aussi conceptuel. De nombreux modèles permettent ainsi de représenter l'organisation des connaissances qui président à l'action des individus (Vidal-Gomel \& Rogalski, 2007). Les notion de concepts en acte (Vergnaud, 1996), de schème (Rabardel, 1995), de concepts pragmatiques (Vidal-Gomel \& Rogalski, 2007), de représentations pour l'action (Weill Fassina, Rabardel, \& Dubois, 1993) permettent ainsi de figurer finement l'organisation des représentations des apprenants, la manière dont ils recueillent et traitent l'information, les indices pertinents qu'ils trouvent dans la réalité, la manière dont ils conçoivent leur action, la mettent en œuvre, l'évaluent et finalement en tirent des apprentissages. Particulièrement utile pour la formation de professionnels évoluant dans des métiers complexes et techniques confrontés à des environnement mouvants (Vidal-Gomel \& Rogalski, 2007), les concepts pragmatiques s'avèrent une notion parlante pour interpréter et comprendre également les actions des apprenants dans divers contextes relationnels (management, délégation, gestion de groupes, ...) qui constituent le centre de préoccupations des psychosociologues. Ces concepts pragmatiques conceptualisent l'objet de l'action, quelle que soit sa nature, et guident à ce titre à la fois la prise d'information sur l'objet et les moyens d'actions. Ils permettent aux personnes d'accéder à une vision simplifiée et opérante d'une réalité complexe. Pouvoir modéliser et représenter ces concepts pragmatiques offre donc une occasion précieuse de favoriser la réflexivité, attitude essentielle pour toute démarche formative intégrant la complexité (Buysse, 2011).

\subsection{La double dimension du travail}

A côté de cet apport réciproque, un premier objet d'échange fondamental réside dans les rapports entre les aspects techniques et psycho-socio-relationnels du travail. En effet, on considère habituellement que le travail contient des aspects que l'on peut qualifier de fonctionnels ou opératoires (les tâches à réaliser) et des aspects relationnels, psychosociaux (les relations humaines qui entourent ces aspects fonctionnels) (Karnas, 2006). Ainsi, on peut dire que l'activité humaine comprend une part d'interaction avec un environnement matériel 
(outils, systèmes opérationnels, matière à transformer, ...) et un environnement psychosocial (collègues, managers, bénéficiaires, clients, ...).

L'importance relative de ces deux dimensions peut être variable : certains métiers techniques solitaires sont dominés par des aspects opératoires, d'autres exigent une interaction permanente avec des collègues, des clients, des usagers. Dans les métiers de la relation humaine (Beckers, 2011), les aspects psychosociaux font en outre partie des aspects fonctionnels, puisque l'objet du travail est la transformation humaine. Le degré de relation à des systèmes techniques et humains, dans le cadre des tâches elles-mêmes ou dans leur contexte, est donc extrêmement variable.

Sur ce point, les deux approches offrent des ressources différentes aux organisations. D'un côté, les approches psychosociales ont développé toute une approche formative de la relation humaine. L'ouvrage de Maisonneuve (2005), dont l'ambition est de faire le bilan de trente années d'apport de la psychosociologie à la formation, montre qu'elle s'avère particulièrement utile à ceux qui, dans l'exercice de leur métier, ont besoin d'appréhender les processus socio-affectifs et socio-opératoires. De l'autre côté, les approches ergonomiques ont développé des outils techniques et conceptuels permettant l'élaboration d'ingénieries de formation qui tiennent compte d'une analyse fine du travail. Les applications aux travaux techniques sont considérables et extrêmement foisonnantes et tout ce courant a contribué à montrer la part de conceptualisation souvent négligée que contient, notamment, le travail manuel (Clot et al., 2005). Cela a aboutit à une diversité de méthodes et techniques qui permettent de passer de l'analyse du travail à l'ingénierie de formation. Il faut toutefois intégrer des nuances importantes: les psychosociologues s'intéressent aux dimensions manuelles et opérationnelles du travail, particulièrement dans le travail des groupes de développement de projets qui concernent des objets techniques et technologiques (Leroy, 1993); tout comme on compte des expériences sur des savoir-être psychosociaux et les métiers qui mettent en relation des humains avec d'autres humains (Pastré, 2005a) dans le domaine ergonomique, par exemple la négociation (Clot et al., 2005), la conduite d'entretiens de face à face (Michel \& Lallemand, 2008), les compétences de directeurs de maisons médicales (Coulet \& Chauvigné, 2005), de conseillers vendeurs (Parage \& Kerverdo, 2008, ...) ou d'enseignants (Veyrac et Boullier, 2011). Mais il est incontestable que certains secteurs d'activité n'ont que peu été abordés par l'analyse de l'activité et la didactique professionnelle (Pastré, 2005a), pendant que d'autres, spécifiquement les travaux manuels, sont restés étrangers à l'approche psychosociologique. Tout travail comportant à la fois des aspects psychorelationnels et des aspects techniques, entendus au sens large, il paraît évident que les apports des deux approches devraient s'avérer complémentaires.

\section{Un échange d'outils et de techniques}

Ayant montré dans les lignes qui précèdent à quel point les deux courants se rejoignaient sur les théories de l'apprentissage, la vision de la construction des connaissances et l'importance de l'action dans cette construction, il y a fort à parier que les outils utilisés par les uns et les autres peuvent s'échanger, s'adapter mutuellement, voire être mobilisés ensemble. On peut ainsi plaider pour un échange technique et méthodologique sur des outils d'apprentissage, et cela dans plusieurs directions. Nous en retiendrons quatre.

1. Les deux approches, à leur manière, construisent des simulateurs de la réalité, notamment lorsque l'objet de la formation est trop complexe à recréer, éthiquement problématique, ou impossible méthodologiquement. Entre les simulateurs techniques créés par les ergonomes 
(Pastré, 2005b) et les simulateurs psychorelationnels des psychosociologues (jeux de rôles (Patin, 2005), trames expérienciées (De Visscher, 2004), mises en situations (Pfeiffer \& Jones, 1983), ...), nombre d'échanges sont possibles. On pourrait également mobiliser conjointement des outils de type simulateurs et de type jeu de rôle pour certaines tâches organisationnelles. La recherche récente de Michel et Lallemand (2008) sur l'apprentissage de la conduite d'entretien ouvre la voie d'une telle approche.

2. Les deux approches sont soucieuses de créer des techniques pour garder des traces de l'activité du sujet dans des situations pouvant se prêter à l'apprentissage, et des façons de construire du savoir à partir de ces traces. Les expériences structurées et grilles d'observation de groupe des psychosociologues, et les différentes techniques d'auto-confrontation, d'interconfrontation, d'instruction au sosie, de débriefing, de confrontation croisée etc. des analystes du travail constituent des ressources à mobiliser en la matière.

3. Les deux approches sont à la recherche d'outils et de méthodes pouvant supporter le processus social d'apprentissage. Les ergonomes auraient avantage à s'intéresser aux principes de l'animation des groupes qui permettent des échanges sur l'expérience entre des participants, les psychosociologues devraient regarder de plus près comment se déroule le processus social dans le cadre, par exemple, des interconfrontations ou de la relation de tutorat.

4. Les deux approches font usage de la vidéo (les ergonomes par différentes techniques pour filmer le travail, les psychosociologues à travers les activités d'autoscopie). Les traitements qui en ressortent sont en revanche assez différents. Ces deux manières de faire se prêtent donc également à des échanges et des mobilisations conjointes.

\section{Le lien entre travail et formation : des stratégies contrastées mais compatibles?}

Enfin, la nature du lien entre le travail et la formation constitue un point de divergence important entre les deux approches, révélateur de manières contrastées de considérer les relations industrielles, et débouchant sur des apports différents au développement des compétences en organisations.

Historiquement, cette question des liens entre travail et formation prolonge une dualité d'enjeux présente depuis le XIXème siècle en formation des adultes (Mucchielli, 2006) : les enjeux d'éducation populaire d'une part et les enjeux de formation professionnelle d'autre part.

D'une part, la vision psychosociologique de la formation est liée depuis ses débuts au développement d'un idéal démocratique, d'une valeur accordée à la participation, à la cohésion et à la justice sociale ainsi qu'au développement de l'individu (Jobert, 2003). Ces conceptions, fortement présentes chez Lewin (Michelot, 2003) ont trouvé un écho dans les concepts d'éducation populaire (Cacéres, 1964), d'animation socioculturelle (Simpson, 1975) ou encore d'éducation permanente (Schwartz, 2001). L'humanisme rogérien, forte source d'inspiration des formateurs psychosociologues (Jobert, 2003) s'inscrit également dans cette vision des choses. L'approche psychosociologique est donc l'héritière de ce courant d'éducation populaire, et historiquement, les psychosociologues ont toujours revendiqué une certaine mise à distance du contexte du travail, y compris dans le cadre de la formation professionnelle, avec l'affirmation forte que celle-ci doit contribuer autant à susciter l'adaptation que la créativité voire la contestation (Lapassade, 1971). Pédagogiquement, l'apprentissage se veut relativement décontextualisé et transversal. Il est vrai que les objets de cet apprentissage, généralement liés au aspects psychorelationnels, se prêtent particulièrement 
bien à une approche moins directement en prise avec le contexte du travail. Les compétences relationnelles ou groupales peuvent en effet plus aisément s'appliquer dans divers contextes que certains gestes opératoires liés à une activité particulière. Dans le courant psychosociologique, les méthodes sont donc davantage analogiques que directement connectées à l'objet d'application: le principe de déstructuration-restructuration (De Visscher, 2001), comme celui de décristallisation (Lewin, 1945), repose en effet sur le postulat d'une certaine distance entre la procédure d'apprentissage et l'environnement d'application. Le refus des solutions toutes faites est affirmé ainsi que l'importance de la créativité (Mucchielli, 2006), le formateur visant plutôt à travailler sur ce qui va amener le changement de comportement (les représentations, les concepts, les attitudes, ...) que sur le changement de comportement lui-même, qui reste aux mains du formé (Delhez, 1999). Cela n'empêche en rien que le transfert sur le terrain soit visé, comme dans l'approche ergonomique, mais c'est à l'aide de situations décadrées (voire « décalées »), puis seulement recadrées, que le formateur vise l'apprentissage, un apprentissage à long terme et fonctionnant sous forme de réminiscence en situations réelles (Faulx, 2008). Les théoriciens de l'apprentissage, dans ce domaine, recommandent d'ailleurs une « juste distance » entre l'objet de l'apprentissage ou son contexte, et la stratégie de formation (Karolewicz, 1998) : trop de recouvrement amène à l'identité, et donc à l'inutilité de la simulation, trop peu de recouvrement amène à l'impossibilité de comparaison (Jaoui, 1980). Cet héritage remonte aux débuts de la démarche groupale. Comme le rappelle Faucheux (1959), pour Lewin, la tâche est un prétexte pour faire fonctionner le groupe. Elle est pensée en termes de motivation et d'apprentissage, non pour un lien direct au travail.

C'est en partie en opposition à cette posture que les ergonomes ont affirmé avec force la nécessité d'un rapprochement de la formation et du travail, et réclamé que l'analyse du travail trouve la place qui lui revient (Parage \& Kerverdo, 2008). Dans cette perspective, Leplat (2005) a stigmatisé les «cas négatifs » de formation qu'il appelle «décontextualisées », notamment les formations qui prennent peu en compte les conditions contextuelles d'utilisation des connaissances communiquées ou apprises. L'importance que les apprentissages soient «situés » (Education Permanente 173 et 174, 2007) est mise en avant, dans la ligne du positionnement de Montmollin (1974) sur la nécessité de construire des formations après analyse des compétences et du comportement au travail des opérateurs expérimentés. Le projet de la didactique professionnelle est à ce sujet on ne peut plus clair : construire des contenus de formation correspondant à la situation professionnelle, et utiliser les situations de travail comme support à la formation (Bouthier, Pastré \& Sammurçay, 1995). L'action formative d'inspiration ergonomique requiert donc de s'intéresser non seulement à l'activité des opérateurs (Clot et al., 2005: Olry, 2008), spécifiquement des opérateurs experts (Zeitler \& Berguerioux, 2008), mais aussi de procéder à l'analyse des situations de travail, ce qui constitue la première étape incontournable en didactique professionnelle (Kunégel, 2005). Cette posture va amener de multiples démarches préalables à la formation telles que la construction de référentiels de compétences, d'emploi et de formation (Coulet \& Chauvigné 2005 ; Breteaudeau \& Cuvilier, 2008 ; Fernagu-Oudet \& Raynaud, 2008), ou l'identification des tâches critiques et des situations-clés (Olry, 2008).

En partageant différents gradients de proximité entre situation de formation et situation de travail, les formateurs des deux courants pourront probablement enrichir leurs stratégies didactiques et développer des ingénieries de formation qui tiennent davantage compte de la diversité des styles d'apprentissage des participants. De manière plus générale, l'enjeu est de déboucher sur un échange, voire une mobilisation conjointe, entre une épistémologie de formation généraliste et décontextualisée, telle qu'on la trouve dans les approches 
psychosociologiques, et une épistémologie de formation spécifique et située, telle que l'a développée l'approche ergonomique.

Néanmoins, le propos doit se nuancer. Les approches psychosociologiques de la formation tiennent également compte des réalités de travail via notamment l'analyse de la demande et des besoins de formation. Les méthodes et approches de formation qui en sont héritées ont largement pénétré le terrain du monde professionnel, et on parle d'ailleurs depuis une quarantaine d'années de «formation psychosociale dans les organisations » (Goguelin et al., 1971). De l'autre côté, les préoccupations humanistes sont présentes dans la formation des adultes d'inspiration psycho-ergonomique, par un travail intense sur les dimensions de santé et de bien-être au travail (Teiger \& Lacomblez, 2005), et par un objectif fondamental qui est de contribuer à un monde professionnel mieux adapté à l'homme et des conditions de travail qui lui sont plus favorables (Bellemare et al., 2001). Il n'en reste pas moins qu'entamer ce dialogue et ce croisement de pratiques constitue une manière aussi de dépasser cette dualité historique des enjeux de la formation, et de rejoindre l'injonction de Merle (2006) qui considère qu'un des enjeux de la Formation Tout au Long de La Vie (FTTLV) est de parvenir à dépasser le dualisme entre humanisme et utilitarisme dans la formation pour bâtir une vision de la formation qui réponde à des enjeux globaux.

\section{Conclusion}

Cette revue critique de la littérature a permis de montrer que les deux courants majeurs dans les pratiques de formation en organisation partagent des filiation théoriques et épistémologiques communes, qu'elles ont déclinées dans des dispositifs, des concepts et des méthodologies très différentes. Il en résulte des approches contrastées, mais compatibles. Leurs apports au développement de la ressource humaine en organisation apparaissent ainsi complémentaires, entre apprentissages transversaux et spécifiques, développement personnel et professionnel, dimensions relationnelles et fonctionnelles du travail. Un tel constat invite les formateurs à intensifier leurs échanges, et les commanditaires à mobiliser les apports conjoints des deux approches afin de développer au mieux les ressources humaines de leurs organisations.

\section{Illustration}

La réalisation de formations combinant les deux approches constitue une entreprise ambitieuse et très stimulante, qui requiert généralement la mise en commun de compétences complémentaires de spécialistes des deux approches. Pour illustrer les fruits d'une telle démarche, nous présentons ci-après un projet de formation développé dans le domaine de la vente en showroom automobile, destiné à des commerciaux dans leur première année d'emploi. Le tableau suit la structure de l'article concernant les apports réciproques.

Tableau 1 : exemple de formation combinant les deux approches

\section{Références}

Abric, J.-C., Doise, W., De Visscher, P., Maisonneuve, J. \& Pain, J. (1999). Dynamique des groupes, analyse institutionnelle et psychologie sociale. Cahiers internationaux de psychologie sociale, 43-44, 7-34. 
Allouche-Benyaoun, J. \& Pariat, M. (1993). La fonction formateur. Paris :

Dunod.

Argyris, C. \& Schön, D. (1974). Theory in Practice : Increasing Professsional Effectiveness. San Francisco : Jossey-Bass

Argyris, C. (1995). Savoir pour agir. Surmonter les obstacles à l'apprentissage organisationnel. Paris, Interéditions,

Astier, P. \& Baroth, M.-C. (2010). Les formateurs face aux changements. Actes du XVIème Congrès de l'Association Internationale de Psychologie du Travail, Lille.

Baiwir, J.\& Delhez, R. (1979). Des exercices structurés en dynamique des groupes : comment ? Cahiers de Psychologie Sociale, 2, 4-8.

Barus-Michel, J., Enriquez, E. \& Lévy, A. (2003). Vocabulaire de psychosociologie. Références et positions. Ramonville Saint-Agne : Erès.

Beckers, J. (2011). Maîtrise de la situation d'enseignement-apprentissage et savoir. Pour une lecture compréhensive des fondements des savoirs professionnels des enseignants. In P. Maubant et S. Martineau (Eds.). Québec : Presses de l’Université.

Bellemare, M., Montreuil, S., Marier, M., Prévost, J. \& Allard, D. (2001). L'amélioration des situations de travail par l'ergonomie participative et la formation. Relation Industrielles / Industrial Relations, 3, 470-490

Bellemare, M., Montreuil, S., Marier, M. Prévost, J. \& Allard, D. (2005). L'implication des acteurs dans l'implantation de projets d'amélioration des situations de travail en ergonomie participative : des constats différenciés dans deux usines. Perspectives Interdisciplinaires Sur le Travail Et la Santé, 6 (2).

Blanchard- Laville C. \& Fablet, D. (2000). L'analyse des pratiques professionnelles. Paris : L'Harmattan.

P.U.F.

Bourgeois, E. \& Nizet, J. (1997). Apprentissage et formation des adultes. Paris :

Bourgeois, E. (2007). Les théories de l'apprentissage. Un peu d'histoire. In E. Bourgeois et G. Chapelle (Eds.). Apprendre et faire apprendre. Paris, P.U.F. pp. 21-38

Bouthier, D., Pastré, P. \& Samurçay, R.. (1995). Le développement des compétences : analyse du travail en didactique professionnelle. Education Permanente, 123, 7-12.

Bretaudeau, J. \& Cuvillier, B. (2008). Approche "didactique professionnelle" sur un métier d'exrtise : le cas de la frappe à froid. Education Permanente, 174, 61-74.

Buysse, A. (2011). Une modélisation des régulations et de la médiation dans la construction des savoirs professionnels de l'enseignant. Pour une lecture compréhensive des fondements des savoirs professionnels des enseignants. In P. Maubant et S. Martineau (Eds.) Québec : Presses de 1'Université. 
Cacérès, B. (1964). Histoire de l'éducation populaire. Paris : Seuil.

Carré, P. \& Caspar, P. (2004). Traité des Sciences et Techniques de la Formation. Paris : Dunod.

Chamberland, G. \& Provost, G. (1996). Jeu, simulation et jeu de rôles. Québec : Presses de l'Université du Québec.

Clot, Y. (2006). Clinique du travail et clinique de l'activité. Connexions, 1, 165-

Clot, Y., Daniellou, F., Jobert, G., Mayen, P. Olry, P. \& Schwartz, Y. (2005). Travail et formation : les bénéfices d'une analyse exigente. Education Permanente, 165, 139160.

Coulet, J.-C. \& Chauvigné, C. (2005). Passer d'un référentiel de compétences à une ingénierie de formation. Education Permanente, 101-114.

De Visscher, P. (1979). Des exercices structurés en dynamique des groupes. Pourquoi ? Les Cahiers de psychologie sociale, $\mathrm{n}^{\circ} 2$, pp. 1-4.

P.U.F.

De Visscher, P. (2001). La dynamique des groupes d'hier à aujourd'hui. Paris :

De Visscher, P. (2004). Les trames expérienciées en dynamique des groupes. Cahiers Internationaux de Psychologie Sociale, 64, 69-70.

Delhez, R. (1999). Réflexions sur une pratique de l'animation. Cahiers internationaux de Psychologie Sociale, 43-44, 35-185.

Doyle, W. 1990. Themes in teacher education researcg ». In W.R. Houston Handbook of research on teacher education. New York : Macmillan, pp. 3-24.

Enriquez, E. (1993). L'approche clinique. Genèse et développement en France et en Europe de l'Ouest. In V. De Gaulejac et S. Roy (Eds.) Sociologies Cliniques. Paris, Desclée de Brouwer.

Faucheux, C. (1959). Kurt Lewin, psychologie dynamique. Les relations humaines. Paris : P.U.F.

Faulx, D. (2008). L'animatique des groupes : esquisse d'un nouveau champ de recherche en formation des adultes. Education Permanente, $n^{\circ} 174,147-156$.

Faulx, D. (sous presse) Un modèle de compétences pour les formateurs d'adultes en organisation ». Psychologie du Travail et des Organisations.

Faulx, D. \& Delvaux, S. (2007). Démarrer une session de formation : réflexions et propositions dans une perspective d'animatique des groupes. Cahiers Internationaux de Psychologie Sociale, 75-76, 157-160.

Falzon, P. \& Teiger, C. (2004). Ergonomie et formation. In P. Carré et P. Caspar (Eds.) Traité des sciences et techniques de la formation. Paris : Dunod, pp. 145-162 
Fernagou Oudet, S. \& Raynaud, B. (2007). Besoin de formation ou développement professionnel ? Créer des occasions de transmission du geste entre bouchers en grande surface. Education Permanente, 174, 89-106.

Fournier, P.-S. (2004). Concevoir une formation: réflexion sur une démarche d'aide à l'apprentissage sur le cours de vie. Perspectives Interdisciplinaires Sur le Travail et la Santé, 6 (2) (revue électronique).

Fournier, P.-S. (2005). Psychologie de la formation, jalons et perspectives par Jacques Leplat. Relations industrielles/Industrial Relations, 60 (2), 375-376.

Goguelin, P. (1991). La formation animation. Une vocation. Paris : ESF

Goguelin, P., Cavozzi, J., Dubost, J. \& Enriquez, E. (1971). La formation psychosociale dans les organisations. Paris : P.U.F.

Gonzalez, R. (2005). L'analyse réflexive collective de l'activité de cadres de direction dans une relation de service "centrée sur autrui" - un cas de formation-action ergonomique dans le secteur des «crèches » municipales en France. Perspectives Interdisciplinaires Sur le Travail Et la Santé, 6 (2). Revue électronique.

Guérin, F., Laville, A. \& Daniellou, F. (1997). Comprendre le travail pour le transformer. Toulouse : Octarès

Hess, R. (1981). La sociologie d'intervention. Paris : P.U.F.

Jaoui, H. (1990). La créativité, mode d'emploi. Paris, : E.S.F.

Jobert, G. (2003). Formation. In J. Barus-Michel, E. Enriquez et A. Lévy (Eds.). Vocabulaire de Psychosociologie. Ramonville Saint Agne, Erès, pp. 353-359.

Karnas, G. (2006). Introduction à la psychologie du travail. Document électronique http://dev.ulb.ac.be/bepsy/cms/index.php L'Harmattan.

Karolewicz, F. (1998). L'expérience. Un potentiel pour apprendre. Paris :

Knowles, M. (1990). L'apprenant adulte. Vers un nouvel art de la formation. Paris : Les Editions d'Organisation.

Kunégel, P. (2005). L'apprentissage en entreprise : l'activité de médiation des tuteurs. Education Permanente, 165, 127-138.

Lamonde Fernande et Sylvie Montreuil. 1995. "Le travail humain, l'ergonomie et les relations industrielles". Relations industrielles/Industrial relations, 50 (4), 695-740.

Landry, S. (2008). Travail, affection, pouvoir dans les groupes restreints. Québec : Presses Universitaires du Québec.

Villars.

Lapassade, G. (1970). Groupes, organisations, institutions, Paris, Gauthier 
Leplat, J. (2005). Les contextes en formation. Education Permanente, 166, 2948.

Leroy, J-F. (1993). La notion de projet. Cahiers Internationaux de Psychologie Sociale, 17, 72-103.

Lewin, K. \& Faucheux, C. (1967). Psychologie dynamique: les relations humaines. Paris, Presses Universitaires de France.

Lewin, K. (1945). Resolving social conflicts. New York Basic Books.

Liétard, B. (2002). Les apprentissages expérientiels : le projet autour de quatre concepts et d'un enterrement. In B. Dauberville (Ed.). Vivre ses projets : construire activement son projet professionnel. Paris : ESF, pp. 51-57

Lindeman, E. (1926). The Meaning of Adult Education. New York : New Republic.

Machin, M. \& Fogarty, G. (2004). Assessing the antecedents of transfer intentions in a training context. International Journal of Training and Development, 8(3), 222-236.

Maisonneuve, J. (2005). Psychosociologie et formation, trente ans de formation relationnelle en groupe. Paris : L'Harmattan.

Marrow A. (1972). Kurt Lewin, sa vie et son æuvre. Paris : E S F.

Meirieu, P. (1987). Groupes et pédagogie. Revue de Psychothérapie Psychanalytique de Groupe, 7-8, 45-58.

Merle, V. (2006). Apprendre tout au long de la vie : pourquoi, comment? Conférence présentée au Premier Forum mondial de l'Education et de la Formation Tout au Long de la Vie. Paris : UNESCO. Document électronique : http://www.centre-inffo.fr/forummondial/spip.php?article63.

Michel, J. \& Lallemand, J. (2008). L'apprentissage de la conduite d'entretien par les formateurs. Travail et Apprentissages, 2, 111-130.

Michelot, C. (2003). « Kurt Lewin ». In J. Barus-Michel, E. Enriquez et A. Lévy (Eds.). Vocabulaire de Psychosociologie. Ramonville Saint Agne : Erès, pp. 505-517

Montmollin, M. (1974). L'analyse du travail, préalable à la formation. Paris : Armand Colin.

Montreuil, S. \& Bellemare, M. (2001). Introduction au numéro spécial Ergonomie, formation et transformation des milieux de travail. Relations industrielles/Industrial Relations, 56 (3), 459-469.

Paris : ESF.

Mucchielli, R. (2006). Les méthodes actives dans la pédagogie des adultes,

Olry, P. \& Parage, P. (2008). Travail et formation: quelques pratiques en émergence. Education Permanente, 174, 5-8. 
Olry, P. (2008). Analyse de l'action et compréhension de l'activité pour la formation. L'exemple des apprentis préposés. Education Permanente, 174, 107-126.

Ombredane, A. \& Faverge, J.-M. (1955). L'analyse du travail. Paris : P.U.F.

Parage P. \& Kervedo, J.-M. (2008). Une démarche d'ingénierie didacique appliquée à une activité de service. Education Permanente, 174, 9-24.

Parage, P. (2008). Retour sur quelques présupposés de base pour l'analyse du travail en vue de la formation. Education Permanente, 174, 127-138.

Pastré, P. (2005a). La deuxième vie de la didactique professionnelle. Education Permanente, 165, 29-46.

Pastré, P. (2005b). Apprendre par la simulation : De l'analyse du travail aux apprentissages professionnel. Toulouse : Octarès.

Pastré, P. (2007). Apprendre à faire. In E. Bourgeois et G. Chappelle (Eds.). Apprendre et faire apprendre. Paris : P.U.F., pp. 109-121.

Pastré, P., Mayen, P. \& Vergnaud, G. (2006). La didactique professionnelle. Revue française de pédagogie, 154, 145-198.

Patin, B. (2005). Le jeu de rôles : pratique de formation pour un public d'adultes. Cahiers Internationaux de Psychologie Sociale, 67-68, 163-178.

Perret-Clermont, A.-N. (1996). La construction de l'intelligence dans l'interaction sociale (cinquième édition). Berne : Peter Lang.

Pfeiffer, W\& Jones, J. (1983). A handbook of structured experiences for human relations (vol I). San Diego : University Associates.

Piskurich, G., Bekschi, P. \& Hall, B. (2000). The ASTD Handbook of Training desingn and delivery. New York : Amacom.

Quadéri, A. (2004). Rhétorique et pragmatique dans les groupes de paroles en gériatrie. Connexions, 82, 49-55.

Rabardel, P. (1995). Les hommes et les technologies. Approche cognitives des instruments contemporains. Paris : Armand Colin.

Rogers, C. (1969). Freedom to learn: A view of what education might become. Columbus $(\mathrm{OH})$ : Merrill.

Saint-Arnaud, Y. (1978). Les petits groupes, participation et communication. Montréal : Presses Universitaires.

Samurçay, R. \& Rabardel, P. (2004). Modèles pour l'analyse de l'activité et des compétences ». In R. Sammurçay et P. Pastré (Eds.). Recherches en didactique professionnelle. Toulouse : Octarès, pp. 163-180.

Schön D. (1994). Le praticien réflexif. A la recherche du savoir caché dans l'agir professionnel. Montréal : Les Editions logiques. 
Shwartz, B. (2001). Education permanente et formation des adultes : évolution des pratiques, évolution des concepts. Education permanente, $n^{\circ} 92$

St-Vincent, M., Lorite, M. \& Chicoine, D. (2001). Participatory Ergonomics Training in the Manufacturing Sector and Ergonomic Analysis Tools. Relations Industrielles / industrial Relations, 56 (3), 491-515.

Teiger, C. \& Lacomblez, M. (2005). L'ergonomie et la trans-formation du travail et/ou des personnes. Education Permanente, 165, 9-28.

Tilman, F. \& Grootaers, D. (2006). Les chemins de la pédagogie. Guide des idéés sur l'éducation, la formation et l'apprentissage. Charleroi : Chroniques Sociales.

Tourraine, A. 1983. La production de la société. Paris : Le Seuil.

Tuckman, B. (1965). Developmental sequence in small groups. Psychological Bulletin, 63, 384-399.

Vergnaud, G. (1996). Savoirs théoriques et savoirs d'action. Paris : P.U.F.

Veyrac, H. \& Bouillier, M.-H. (2011). In P. Maubant et S. Martineau (Eds.)Les concepts de représentations de la tâche en ergonomie pour l'analyse des fondements des pratiques professionnelles des enseignants. Pour une lecture compréhensive des fondements des savoirs professionnels des enseignants.. Québec : Presses de l'Université.

Vidal, J.-P. (2006). Analyse clinique de conduites professionnelles: une méthodologie originale pour un travail en groupe. Connexions, 86, 65-96

Vidal-Gomel, C. \& Rogalski. J. (2007). La conceptualisation et la place des concepts pragmatiques dans l'activité professionnelle et le développement des compétences. @ ctivité, 4 (1). Revue électronique.

Voisin, A. (2004). Ergonomie et formation. In P. Carré et P. Caspar (Eds.). Traité des Sciences et techniques de la formation continue. Paris : Dunod, pp. 84-102.

Weill-Fassina, A., Rabardel, P. \& Dubois, D. (Eds.) (1993). Représentations pour l'action. Toulouse : Octarès.

Wenger E. (1998). Communities of Practice: Learning, Meaning, and Identity. Cambridge University Press.

Zeitler, A. \& Bergerioux, L. (2008). Former des experts, une mission impossible ? Education Permanente, 174, 25-37. 
